\title{
High-performance GPCR optogenetics based on molecular properties of animal opsins, MosOpn3 and LamPP
}

Mitsumasa Koyanagi ${ }^{1,2,4, *}$, Baoguo Shen ${ }^{1,4}$, Takashi Nagata $^{1}$, Lanfang Sun $^{1}$, Seiji Wada ${ }^{1}$, Satomi Kamimura ${ }^{1}$, Eriko Kage-Nakadai ${ }^{2,3}$, and Akihisa Terakita ${ }^{1,2, *}$

${ }^{1}$ Department of Biology and Geosciences, Graduate School of Science, Osaka City

University, 3-3-138 Sugimoto, Sumiyoshi-ku, Osaka 558-8585, Japan.

${ }^{2}$ The OCU Advanced Research Institute for Natural Science and Technology, Osaka City

University, 3-3-138 Sugimoto, Sumiyoshi-ku, Osaka 558-8585, Japan.

${ }^{3}$ Graduate School of Human Life Science, Osaka City University, 3-3-138 Sugimoto, Sumiyoshi-ku, Osaka 558-8585, Japan.

${ }^{4}$ These authors contributed equally.

*To whom correspondence should be addressed. E-mail: koyanagi@ osaka-cu.ac.jp, terakita@osaka-cu.ac.jp 


\section{Abstract}

Optogenetics for GPCR signaling is highly valuable but still requires effective and versatile tools with performance evaluation from molecular properties. Here we investigated performance of two animal opsins, mosquito Opn3 (MosOpn3) and lamprey parapinopsin (LamPP) in optical manipulation in vivo by using C. elegans. MosOpn3 introduced in a nociceptor neurons induced avoidance responses light-dependently with a retinal isomer ubiquitously present in every tissue, like $\mathrm{ChR} 2$ and unlike canonical vertebrate opsins. Remarkably, the sensitivity is $\sim 7000$ times higher than the case of ChR2 in the light-induced behavior. LamPP introduced in motor neurons induced violet light-dependent stop and green light-dependent go, demonstrating color-dependent manipulation of behaviors using LamPP. Furthermore, our molecular engineering extended the usability of MosOpn3 and LamPP to different signaling cascades and kinetics. Current findings demonstrated that the availability of two animal opsins is equivalent to that of $\mathrm{ChR} 2$ in terms of retinal requirement, providing solid strategies for GPCR optogenetics. 


\section{Introduction}

G protein-coupled receptors (GPCRs) are transmembrane receptors, which are involved in various cellular and physiological functions including neural responses, cell metabolisms, and hormonal responses ${ }^{1,2}$. GPCRs generally bind a variety of chemical ligands such as odorants, hormones and neurotransmitters, and the extracellular signals are transduced into intracellular signaling (GPCR signaling) via heterotrimeric G proteins. The GPCR signaling varies mainly depending on the subtype of $G$ protein alpha subunit and includes cAMP-signaling up- and down-regulated by Gs- and Gi-type G proteins, respectively, and phosphoinositol-signaling for $\mathrm{Ca}^{2+}$ elevation mediated by Gq-type $\mathrm{G}$ protein. Most animals have hundreds of GPCR genes and human, for example, has $~ 800$ GPCR genes, indicating the importance of GPCR signaling for biological activities ${ }^{3}$. Although structure-function relationships of many GPCRs have been well investigated so far, for comprehensive understanding of GPCR-based physiologies as well as controlling them precisely, optical manipulation of GPCR signaling would be one of the ultimate approaches because of high temporal resolution of light stimulus.

Animal rhodopsins (opsin-based pigments), which underlie vision and non-visual functions such as circadian photoentrainment in varied animals, consist of a protein moiety, opsin, and 11-cis retinal as a chromophore in many cases and basically serve as 
light-sensitive GPCRs ${ }^{4}$. Therefore, opsins have been considered promising tools for optical manipulation of GPCR signaling, and indeed, such optogenetic application has succeeded to some extent using vertebrate visual opsins ${ }^{5-7}$. However, the fact that only a small number of papers on optogenetic research using animal opsins have been published until now compared to those using microbial rhodopsins such as ChR2 for optical manipulation of neural activities suggests that there is still room for improvement. The point would be related to molecular properties of vertebrate visual opsins; 1) they basically form photopigments by binding to 11-cis retinal, which is abundant in photoreceptor tissues like eyes, but not in other tissues (11-cis-retinal-requirement), and 2) after light absorption their photopigments immediately release chromophore (bleach) to be functionless (bleaching property), both of which are unfavorable characteristics for high performance optical manipulation of GPCR signaling, especially in vivo ${ }^{4}$. To overcome the problems associated with the molecular properties, utilization of animal opsins that is more suitable for optogenetic tools should be required.

To date, thousands of opsins have been identified from a wide range of animals from cnidarians to vertebrates, and they are phylogenetically divided into eight or more groups, which is almost consistent with the classification based on $G$ protein selectivity and activation manner ${ }^{4,8}$. With respect to the photochemical property, opsins are basically 
classified into two types, bleaching opsins like vertebrate visual opsins and

bleach-resistant or bistable opsins, which convert to stable active states upon light absorption and in many cases, active states revert to the original inactive state by subsequence light absorption ${ }^{4,9}$, like invertebrate visual opsins ${ }^{10-13}$. The bistable nature appears to be suitable for sustained optical manipulation of GPCR signaling. In fact, a bistable opsin melanopsin (OPN4) was applied to optical control of some physiologies including restoration of vision ${ }^{14-16}$. In addition to melanopsin, we have identified many kinds of bleach-resistant/bistable opsins from both invertebrates and vertebrates ${ }^{10,17-23}$.

Among them, we particularly focused on optogenetic potentials of Opn3 and parapinopsin because of their interesting molecular properties, which are recently receiving increasing attention $^{24}$.

Opn3 was first identified from mammalian brain and therefore originally called encephalopsin ${ }^{25}$. Then its homologs were identified from many animals and revealed to be expressed in their various tissues including brain, suggesting their involvement in photoreception in "non-photoreceptive" tissues. We have previously succeeded in functional analyses of members of the Opn3 group ${ }^{22,26}$ and found that one of the members, mosquito Opn3 has a unique property; it forms a photopigment that light-dependently activate Gi and Go-type G protein, when bound to 13-cis retinal as well as 11-cis 
retinal $^{22,27}$. Since 13 -cis retinal is thermally equilibrated with all-trans retinal, a retinal isomer ubiquitously present in animals, we have proposed the idea that the Opn3 is applicable to anywhere in the body as an optogenetic tool. In fact, mammalian cultured cells expressing the mosquito Opn3 exhibited light-induced decrease of intracellular cAMP level probably mediated by the Gi activation with all-trans retinal addition or even without retinal addition, under the presence of only a small amount of retinoid in serum ${ }^{22}$. The idea was also supported by the recent report showing optogenetic silencing of neurotransmitter release with the mosquito Opn3 in vitro and in vivo ${ }^{28}$.

Another promising bistable opsin parapinopsin was first identified from catfish pineal and parapineal organs ${ }^{29}$ and thereafter from many lower vertebrate pineal-related $\operatorname{organs}^{17,23,30,31}$. Spectroscopic and biochemical analyses revealed that the lamprey parapinopsin is a UV-sensitive bistable opsin, which activates transducin and Gi-type G protein upon UV-light absorption ${ }^{32-34}$. Notably, parapinopsins convert to the stable active state having an absorption maximum at $\sim 500 \mathrm{~nm}$, in green region, which is largely distinct from that of the inactive dark state ${ }^{32}$. The large spectral difference between the inactive and active states allows selective illumination of active state, resulting in its complete recovery to the inactive state ${ }^{17}$. The same is true for the signal transduction level. The $G$ protein activation by parapinopsin were up- and down-regulated by UV and green light 
illumination, respectively in vitro and in vivo ${ }^{33-38}$, demonstrating its optogenetic potential for color-dependent on and off of GPCR signaling.

Here, to evaluate performances of the mosquito Opn3 (MosOpn3) and lamprey parapinopsin (LamPP) in optical manipulation of GPCR signaling in vivo based on their molecular properties, we focused on Caenorhabditis elegans, in which relationships between GPCR signaling and behaviors have been well defined. Importantly, in the case of optogenetics in C. elegans, isomeric forms of chromophore retinal can be controlled by exogenously adding specific retinal isomers ${ }^{6,39}$, which is an irreplaceable advantage in testing the chromophore requirement of opsin for functioning in vivo, in 11-cis form poor condition. In this paper, we showed that MosOpn3 functions in vivo under the absence of 11-cis retinal with much higher sensitivity compared to ChR2, which is the most used optogenetic tool. We also succeeded in color-dependent manipulation of a C. elegans behavior by using LamPP. Together with our demonstration of introducing G protein selectivity of particular GPCRs into MosOpn3 and LamPP, current findings provide versatile and powerful optogenetic tools for manipulating GPCR signaling and various physiologies based on the molecular properties of the two opsins. 


\section{Results}

\section{Optical manipulation of $C$. elegans behavior using the mosquito Opn3}

We evaluated the performance of mosquito Opn3 (MosOpn3) for its molecular property-based optical manipulation of GPCR signaling in vivo using C. elegans. Since MosOpn3 is a Gi/o-coupled opsin ${ }^{22}$, we focused on ASH neurons, a kind of nociceptors, in which chemoreceptors trigger Gi/o-like G protein (ODR-3)-mediated signal transduction upon ligand binding to induce avoidance behavior of C. elegans ${ }^{40,41}$ (Fig. 1a). MosOpn3 was introduced into ASH neurons under the control of the promoter of sra-6, a chemosensory receptor mainly expressed in ASH neurons ${ }^{42}$ (Fig. 1b). We obtained several lines of transgenic ( $\mathrm{Tg}$ ) worms that express MosOpn3 in ASH neurons (MosOpn3-worms) with the aid of the pharynx expression of mCherry introduced as a selection marker together with MosOpn3. The expression of MosOpn3 in ASH neurons were confirmed by the expression of GFP, which was designed to be bicistronically expressed with MosOpn3 in ASH neurons. We performed behavioral experiments (Fig. 1c) for a light-induced avoidance of MosOpn3-worms that were fed 11-cis retinal-containing Escherichia coli (MosOpn3/11-worms). As results, MosOpn3/11-worms exhibited clear avoidance responses by illumination of white light (Fig. 2a upper panels, Supplementary Movie 1a).

On the other hand, MosOpn3-worms without a supply of retinal 
(MosOpn3/NoRet-worms) did not exhibit the light-induced avoidance responses at all

(Supplementary Movie 1b), which is consistent with previous observations that the addition of retinal is required for functioning of rhodopsins in C. elegans ${ }^{6,39}$. The retinal-

requirement demonstrated that the light-induced avoidance responses of MosOpn3-worms

were not caused by the endogenous light sensor protein, lite- $1^{43,44}$, but indeed by

MosOpn3 provably through ODR-3-mediated signaling. Collectively, the results ensure

the validity of our experimental conditions including light intensity for investigating the

functionality of heterologously expressed animal opsins in C. elegans. Importantly, when

MosOpn3-worms were fed all-trans retinal (MosOpn3/AT-worms), they also exhibited

the light-induced avoidance responses (Fig. 2a lower panels, Supplementary Movie 1c)

like the case of MosOpn3/11-worms, which can be explained by the unique molecular

property of MosOpn3, the pigment formation ability with 13-cis retinal thermally

generated from all-trans form as observed in our previous in vitro experiment ${ }^{22}$.

\section{Comparison of the functionality and efficiency in vivo between MosOpn3 and BovRh}

To evaluate the advantage of MosOpn3 in optical manipulation of GPCR signaling in vivo, we also investigated the light-induced avoidance behaviors of $\mathrm{Tg} C$. elegans expressing a bleaching opsin, bovine rhodopsin (BovRh), which activates Gi/o type $\mathrm{G}$ protein ${ }^{45}$ like 
MosOpn3 but does not bind 13-cis retinal or all-trans retinal unlike MosOpn3. Tg worms established to express BovRh in ASH neurons (BovRh-worms) exhibited the avoidance behavior by white light illumination when they were fed 11-cis retinal-containing E. coli (Supplementary Movie 1d, e). On the other hand, BovRh-worms did not respond to light when they were fed all-trans retinal-containing E. coli (Supplementary Movie 1f), which is different from the case of MosOpn3/AT-worms, showing good agreement with molecular properties of respective opsins. We then compared the performances of these two animal opsins in optical manipulation of GPCR signaling in vivo quantitatively. We investigated relationships between light intensity and light-induced avoidance response probabilities of MosOpn3- and BovRh-worms with various amounts of 11-cis retinal to find the necessary amount of 11-cis retinal for functioning in ASH neurons to avoid side effects caused by an excess amount of retinal. The light intensity-response probability relationships revealed that MosOpn3-worms exhibited a similar light sensitivity even when the amount of 11-cis retinal was reduced to $1 / 100$, and the sensitivity was slightly decreased under 1/1000 amount of 11-cis retinal (Supplementary Fig. 1a). In the case of BovRh-worms, the light sensitivity was also similar even when the amount of 11-cis retinal was reduced to $1 / 100$, but the sensitivity was largely decreased under $1 / 1000$ amount of 11-cis retinal (Supplementary Fig. 1b). These results indicate that the 1/100 
amount is necessary to induce maximum performance for both opsins in this behavior. We then compared performances of MosOpn3- and BovRh-worms in the presence of the minimum necessary amount of retinal. We found that light sensitivities of MosOpn3/11and BovRh/11-worms are similar, which suggests a similarity in performance of 11-cis binding-MosOpn3 and BovRh in the ASH neurons (Fig. 2b). On the other hand, in the same amount of all-trans retinal, MosOpn3/AT-worms exhibited the avoidance responses by illuminations of $1 / 10000 \sim 1 / 1000$ of the maximum intensity $\left(\mathrm{I}_{0}\right)$ of light, whereas BovRh/AT-worms did not exhibit any avoidance responses even by illuminations of the maximum intensity of light (Fig. 2c), demonstrating that the sensitivity of MosOpn3/AT-worms is more than 1000 times higher than that of BovRh/AT-worms. Remarkably, the sensitivity of MosOpn3/AT-worms is comparable to that MosOpn3/11-worms (Fig. 2c). In addition, when the amounts of 11-cis and all-trans retinal added to worms were reduced to 100 times lower level, the sensitivities of MosOpn3-worms under the presence of 11-cis and all-trans retinal decreased similarly (Fig. 2d, e). Since the decreases of sensitivity are explained by those of formed photopigment amount in C. elegans, the similarities in sensitivity between MosOpn3/11and MosOpn3/AT-worms under varied retinal amounts suggest that MosOpn3 bound to 11-cis retinal and MosOpn3 bound to 13-cis retinal, which is thermally generated from 
all-trans retinal, can function with similar efficiency in ASH neurons. These results clearly demonstrated that MosOpn3 performs efficiently in ASH neurons to evoke avoidance responses even in the presence of all-trans retinal like microbial rhodopsins.

\section{Engineering MosOpn3 for light-dependent upregulation of intracellular cAMP and}

\section{$\mathrm{Ca}^{2+}$ levels}

MosOpn3 forms a photopigment by binding 13-cis retinal to activate Gi and Go type G protein in a light dependent manner ${ }^{22}$, which leads to decrease of cAMP. To expand the scope of optogenetic application of MosOpn3, we engineered MosOpn3 to upregulate cAMP and $\mathrm{Ca}^{2+}$ levels, through Gs- or Gq-type $\mathrm{G}$ protein, respectively by exchanging cytoplasmic region(s) including the third cytoplasmic region, the major determinant of $\mathrm{G}$ protein selectivity for Class A GPCRs ${ }^{45-51}$. We chose the 32 -adrenergic receptor (B2AR) and $\alpha 1$-adrenergic receptor ( $\alpha 1 \mathrm{AR})$, which are generally considered to selectively activate Gs- and Gq-type G protein, respectively, as donor GPCRs. We generated a series of MosOpn3-ß2AR and MosOpn3- $\alpha 1$ AR chimeras, in which cytoplasmic region(s) were replaced with those of $32 \mathrm{AR}$ or $\alpha 1 \mathrm{AR}$ according to previous reports ${ }^{5,50}$, and investigated functional conversion. In the case of MosOpn3-ß2AR chimeras, we measured light-induced increases of cAMP level in cultured cells expressing each chimera using the 
GloSensor cAMP assay, and revealed that all chimeras induced obvious cAMP increases light dependently in contrast to the cAMP decrease in the wild type (WT) even without addition of retinal (Fig. 3a), indicating successful conversion of cAMP regulation from down- to up-regulation by MosOpn3 bound to endogenous retinal in the culture medium. Any MosOpn3-B2AR chimeras exhibited larger cAMP increases upon light absorption than the previously reported MosOpn3 chimera containing the third cytoplasmic region of jellyfish Gs-coupled opsin ${ }^{20,26}$. Among all, the chimera in which all cytoplasmic regions were replaced with those of $ß 2 \mathrm{AR}$ (MosOpn3-ß2ARiL123C) exhibited the largest cAMP increase and the chimera containing the second, third and C-terminal region of $\beta 2 \mathrm{AR}$ (MosOpn3-32ARiL23C) was comparable. An interesting finding is that introducing the first cytoplasmic region of $\beta 2 \mathrm{AR}$ accelerates the reversion of increased cAMP levels to the basal level, which provides choices of different kinetics of cAMP changes based on the chimera species (Fig. 3a).

Regarding the regulation of $\mathrm{Ca}^{2+}$ level, aequorin luminescence-based calcium assay revealed that MosOpn3 WT originally exhibited a light-induced increase in cultured cells (Fig. 3b), although it also induced a decrease of cAMP level as described previously ${ }^{22}$ (Fig. 3a, b). Then we evaluated specific regulation of $\mathrm{Ca}^{2+}$ by MosOpn3- $\alpha 1 \mathrm{AR}$ chimeras in cultured cells expressing each chimera. Contrary to the case of MosOpn3-B2AR 
chimeras, only the MosOpn3- $\alpha 1$ AR chimera having the third cytoplasmic region of $\alpha 1 \mathrm{AR}$ (MosOpn3- $\alpha 1$ ARiL3) exhibited a light-induced $\mathrm{Ca}^{2+}$ increase under the condition without 11-cis retinal addition, although the $\mathrm{Ca}^{2+}$ increase by the chimera is less than that by the WT and the chimera still exhibited cAMP decrease (Fig. 3b). To improve the specificity of the MosOpn3- $\alpha 1$ ARiL3 for $\mathrm{Ca}^{2+}$ increase, we further engineered the chimera by fine-tuning of the third cytoplasmic region to be exchanged by shifting the donor/acceptor boundaries by two or four amino acids. In all possible combinations, we found that chimeras generated by reduction of two amino acids at the N-terminal and addition of four amino acids at $\mathrm{C}$-terminal of the third cytoplasmic loop of $\alpha 1 \mathrm{AR}$

(MosOpn3- $\alpha 1$ ARiL3(-2/+4)) exhibited the highest functionality in light-induced $\mathrm{Ca}^{2+}$ elevation, which is $\sim 2.5$-fold higher than the case of WT without cAMP decrease. The light-induced cAMP increases observed in the case of MosOpn3- $\alpha 1 \mathrm{ARiL3}(-2 /+4)$ and MosOpn3- $\alpha$ 1 ARiL3(-2/+2) were presumably caused by $\mathrm{Ca}^{2+}$ elevation $^{52-54}$. These results suggest the practical strategy for creating on-demand optogenetic tools based on MosOpn3 for manipulating intracellular cAMP and $\mathrm{Ca} 2+$ levels probably through optimizing $\mathrm{G}$ protein signaling preference.

\section{Parapinopsin for color-dependent manipulation of GPCR signaling}


In cases of visible light-sensitive bistable opsins including MosOpn3, the dark (inactive) and photoproduct (active) states have largely overlapped absorption spectra in the visible light region. On the contrary, in the case of parapinopsin, the absorption spectra of UV-sensitive inactive state and visible light sensitive active state are distinct from each other, enabling to illuminate only the active state with visible light, which leads to complete regeneration of inactive state. To evaluate a contribution of the photoregeneration ability in optical manipulation of GPCR signaling in vivo, we focused on another $C$. elegans behaviors, coordinated movement regulated by acetylcholine. Using the promoter of unc-17, we introduced LamPP into cholinergic motor neurons, in which activation of Go-mediated signal transduction down-regulates acetylcholine releases to lead uncoordinated movement including coiling of C. elegans ${ }^{55,56}$ (Fig. 4a, b). Tg worms expressing LamPP in cholinergic motor neurons (LamPP-worms) were obtained with the same procedure as that for MosOpn3 worms. When we illuminated LamPP-worms fed 11-cis retinal-containing $E$. coli with violet light, the Tg worms stopped moving and coiled as expected, and upon subsequent green light illumination, the Tg worms restarted coordinated movement (Fig. 4c, d, Supplementary Movie 2). The violet light-induced coiling of LamPP-worms sustained for 30 min after the turn-off of light, and subsequent green light illumination restored the movement (Fig. 4e). The behavioral switching 
between coiling and moving by violet and green light stimuli occurred repeatedly

(Supplementary Movie 2), demonstrating that the C. elegans behaviors are manipulated in a color-dependent manner using LamPP. In other words, the result suggested that introducing LamPP into cells containing Gi/o-mediated signaling could render physiologies color-dependency. Furthermore, LamPP-worms fed all-trans retinal under the red light $(600 \mathrm{~nm})$ exhibited violet-light-induced stop and green-light-induced recovery like the case of LamPP-worms fed 11-cis retinal (Fig. 4d). Since retinal hardly absorbs red light, the phenomena can be explained by the complete photoregeneration ability of LamPP; LamPP bound all-trans retinal to form the active state (LamPP* in Fig. 4b) directly and the active state completely converted to the 11-cis retinal-binding inactive state by red light absorption.

Again, we engineered LamPP, which originally activates Gi/o-type G protein, to activate Gs-type G protein to up- and down-regulate intracellular cAMP levels with violet and visible light absorption, respectively, which is in the opposite direction caused by the WT. We generated a series of LamPP chimeras, in which cytoplasmic region(s), including the third cytoplasmic region was replaced with those of $\beta 2$-adrenergic receptor as in the case of engineering Gs-coupled MosOpn3. Light-induced changes of cAMP level in cultured cells expressing each chimera were measured using the GloSensor cAMP assay. 
We found that only the cells expressing the chimera containing the third cytoplasmic loop alone (LamPP-ß2ARiL3) exhibited a significant violet light-induced increase of cAMP level, which in turn decreased to the basal level by subsequent green-light illumination (Fig. 5a). The color-dependent up- and down-regulations of cAMP levels occurred repeatedly. Notably, the cAMP increases induced by activation of the chimera were composed of two phases; upon violet-light illumination, cAMP levels rapidly increased to a higher level and immediately decreased to a lower level (acute phase) and the lower level sustained for more than 1 hour until green light illumination (chronic phase). Interestingly, when LamPP-ß2ARiL3-expressing cells were illuminated with blue light, the cAMP level was set at a level between levels caused by violet and green light illumination (Fig. 5b). Furthermore, the cAMP levels caused by blue light illumination were almost constant regardless of the levels just before illumination. The color dependency of sustained cAMP level could be explained in part by color dependent photoequilibrium of the inactive and active states of LamPP ${ }^{17,35}$. We also tested the performance of an additional LamPP chimera containing the third cytoplasmic region of the jellyfish Gs-coupled opsin, LamPP-JelOpiL3. The LamPP-JelOpiL3 exhibited much higher amplitude of violet light-induced increases compared with LamPP-B2ARiL3, whereas the increased cAMP level gradually decreased (Supplementary Fig. 2), unlike the 
case of LamPP-B2ARiL3. The increase and decrease of cAMP level by violet light and green light illumination also occurred repeatedly for LamPP-JelOpiL3. Collectively, these Gs-coupled LamPPs enable to manipulate Gs-mediated signal transduction in a color dependent manner, showing successful expansion of LamPP as tools for color-dependent manipulation of GPCR signaling. 


\section{Discussion}

In this study, we demonstrated high performances of two bistable opsins, mosquito Opn3

(MosOpn3) and lamprey parapinopsin (LamPP) in manipulating GPCR signalings in vivo.

Tg-worms expressing MosOpn3 in ASH neurons exhibited light-induced avoidance

behaviors under the presence of 11-cis retinal and all-trans retinal with a similar

sensitivity, which is comparable to the case of $\mathrm{Tg}$-worms expressing bovine rhodopsin

(BovRh) under the presence of 11-cis retinal (Fig. 1, 2). On the other hand, BovRh-worms

did not exhibit light-induced avoidance behaviors under the presence of all-trans retinal as

shown in the previous report examining the functionality of BovRh in other type of

neurons of $C$. elegans, in which 9-cis retinal addition induced light-dependent neural

responses but all-trans retinal addition did not ${ }^{6}$. Since all-trans retinal is basically present

in every tissue, the perfomance of MosOpn3 under the presence of all-trans retinal

comparable with that of BovRh under the 11-cis retinal suggests that MosOpn3 has a

crucial advantage in optical manipulation of GPCR signaling in vivo. It is widely accepted

that microbial rhodopsins such as ChR2 are powerful tools for use in optogenetics,

especially in optical manipulation of neural activities ${ }^{57-60}$. One of the reasons for the

popularity is that microbial rhodopsins form photosensitive pigments by binding all-trans

retinal to function in every tissue, and the 11-cis retinal-requirement of bleaching opsins is 
the main reason for unpopularity of animal opsins. In terms of this point, the current study demonstrated that the availability of MosOpn3 could be equivalent to that of microbial rhodopsins. Moreover, the sensitivity of the avoidance response light-dependently induced by MosOpn3 in ASH neurons was revealed to be $\sim 0.0002 \mathrm{~mW} / \mathrm{mm}^{2}$ (white light) under the presence of all-trans retinal (Fig. 2c), which is $\sim 7000$ times higher than that induced by ChR2 in ASH neurons $\left(\sim 1.48 \mathrm{~mW} / \mathrm{mm}^{2}\right.$, blue light) reported in the previous study ${ }^{61}$. In fact, we generated $\mathrm{Tg}$ worms expressing $\mathrm{ChR} 2$ in $\mathrm{ASH}$ neurons and tested light-induced avoidance behaviors with the same protocol as in cases of MosOpn3 and BovRh but no reproducible avoidance response was observed by illuminating with $\sim 0.8 \mathrm{~mW} / \mathrm{mm}^{2}$ of white light, the highest light intensity of our light source. These data demonstrated that MosOpn3 could function as a light-sensitive switch to provide much higher photosensitivity to any tissue with much lower light-induced toxicity and/or temperature increase compared with the case of $\mathrm{ChR} 2$. There was a potential concern that the basal activity of 13-cis retinal-bearing MosOpn3 pigment might affect cellular conditions intrinsically in vivo because the activity of $\mathrm{G}$ protein by the 13-cis retinal-bearing pigment in the dark was higher than that of the 11-cis retinal-bearing pigment ${ }^{22}$. However, MosOpn3-worms fed all-trans retinal-containing E. coli, in which 13-cis retinal-bearing MosOpn3 pigments formed, normally behaved in the dark (Fig. 2). The data suggests that 
intracellular conditions in ASH neurons were somehow maintained to be normal, even with the partial $\mathrm{G}$ protein activation by 13-cis retinal-bearing MosOpn3 pigment. Then we established B2AR (Gs-coupled receptor) and $\alpha 1 \mathrm{AR}$ (Gq-coupled receptor) versions of MosOpn3 by replacing cytoplasmic region(s) with those of $\beta 2 \mathrm{AR}$ and $\alpha 1 \mathrm{AR}$, respectively, to expand the applicability of MosOpn3 (Fig. 3). Analysis of series of MosOpn3 chimeras revealed that fine tuning of replaced regions improves the $\mathrm{G}$ protein activation efficiency and specificity ( $\alpha 1 \mathrm{ARiL3})$ and alters the kinetics of chimeras ( $(32 \mathrm{AR})$, which could be a guide for making various MosOpn3 chimeras with other GPCRs of interest for appropriate purposes.

We also demonstrated color-dependent manipulation of GPCR signaling by LamPP to lead behavioral switching between coiled and moving of $C$. elegans by violet and green light stimuli, respectively (Fig. 4d, e). The switching is consistent with the molecular behavior of LamPP by UV and visible light absorption ${ }^{17}$, which suggests that the photoregeneration ability of LamPP contributes to the mode change of animal behaviors. Interestingly, we recently reported that in the zebrafish pineal photoreceptor cells, the parapinopsin alone generates color opponency; UV and orange lights induce hyperpolarization and depolarization, respectively, based on the photoequilibrium levels between inactive and active states of the parapinop $\sin ^{35}$. The idea was also supported by 
current results by heterologous expressions of LamPP in C. elegans neurons, showing that introducing LamPP is sufficient for color-dependent behavioral switching. Furthermore, we demonstrated that LamPP-worms fed all-trans retinal under the red light $(600 \mathrm{~nm})$ responded to light stimuli (Fig. 4e), indicating that LamPP could function as a light-sensitive switch in every tissue with red light pre-illumination. The availability of LamPP is practically equivalent to MosOpn3 as well as microbial rhodopsins.

The color-dependent manipulation of Gi/o type G protein by the wild type LamPP has also been expanded to that of Gs type G protein by LamPP-BARiL3 and LamPP-JelOpiL3, which enables color-dependent manipulation of intracellular cAMP levels in the reverse direction to the reactions by the wild type (Fig. 5). Interestingly, LamPP-BARiL3 showed molecular properties for not only color-dependent switch-on and switch-off of GPCR signaling but also color-dependent and context-independent maintenance of intracellular cAMP level (Fig. 5). The results indicate that LamPP-ßARiL3 achieves clamping intracellular cAMP levels depending on color of light.

We also emphasize that the cAMP levels were maintained in the DARK after a light flash, depending on its color, suggesting an alternative strategy for "light"-regulation of cAMP level. On the other hand, the "cAMP-clamping" was not achieved in the case of LamPP-JelOpiL3 (Supplementary Fig. 2). The clamped cAMP level could relate to shut 
off manners of the active state of LamPP-BARiL3 based on unknown effects of the third cytoplasmic loop of $32 \mathrm{AR}$ including phosphorylation and/or arrestin-binding, which provides a new insight into prolongation of GPCR signalings.

In this study, the availability of two bistable animal opsins has shown to be equivalent to that of microbial rhodopsins including $\mathrm{ChR} 2$ in terms of retinal requirement, which would accelerate research by optical manipulation of GPCR signaling. In manipulating GPCR signaling, chemical manipulation, namely chemogenetics using chemoreceptors such as DREADD (designer receptor exclusively activated by designer drugs) have been widely used ${ }^{62-64}$, which is in contrast to the situation of optogenetics with animal opsins, mainly bleaching opsins. The low popularity of optical manipulation of GPCR signaling was mainly due to the absence of effective and robust tools but the situation has been recently changing with bistable opsins, especially MosOpn3 and LamPP ${ }^{28,36-38}$, and advantages of them and their derivatives were systematically demonstrated based on their molecular properties in this paper. The main advantage of chemogenetics is that stimuli (chemicals) can be reliably delivered deep inside the body. On the other hand, temporally precise manipulation of GPCR signaling in vivo is basically unachievable by chemogenetics but achievable by optogenetics. In particular, temporally precise termination of $\mathrm{G}$ protein signaling as demonstrated by LamPP (Fig. 5) is a 
significant advantage of optogenetics, which enables controlling durations, intervals and numbers of the stimuli. Together with chemical manipulation, the optical manipulation with bistable animal opsins would greatly facilitate comprehensive and deeper understating of GPCR-based physiologies as well as GPCR signaling themselves. 


\section{Materials and Methods}

Generating Tg C. elegans. The 2.4 kbp promoter sequence of $s r a-6^{42}$ was obtained from the C. elegans genomic DNA by PCR. The $3.3 \mathrm{kbp}$ promoter sequence of unc-17 was a generous gift from Prof. Robert Lucas ${ }^{56}$. The vector backbone containing SL1 and GFP was obtained by digestion of the plasmid [pEM1 = flp-21::LoxPStopLoxP::npr-1 SL2

GFP] (Addgene plasmid \# 24033) ${ }^{65}$ with NotI and KpnI. The sra-6 promoter linked with MosOpn3 cDNA or unc-17 promoter linked with LamPP cDNA was introduced into the vector. Each plasmid was co-injected into the wild type C. elegans strain N2 obtained from the Caenorhabditis Genetics Center with pmyo-2::mCherry as a selection marker. The wild and Tg strains (psra-6::MosOpn3::GFP, punc-17::LamPP::GFP) were cultured according to standard methods ${ }^{66}$.

\section{Behavioral experiments}

Adult worms were used for behavioral experiments. Tg worms were fed Escherichia coli strain OP50 which are mixed with or without certain amounts of retinal at $24 \mathrm{~h}$ before experiments. The standard concentrations of 11-cis retinal or all-trans retinal in OP50 for our experiment were $0.38 \mathrm{mM}$ or $0.67 \mathrm{mM}$, respectively, and diluted to $1 / 100$ to $1 / 10000$. Worms were monitored under infrared light illumination. White lights $\left(\mathrm{I}_{0}=\sim 0.8\right.$ 
$\mathrm{mW} / \mathrm{mm}^{2}$ ) supplied by a $200 \mathrm{~W}$ metal-halide lamp (PhotoFluor II, 89 North) with or without ND filter(s) were applied as light stimuli for MosOpn3-worms. Narrow band violet $(410 \mathrm{~nm})$ and green $(510 \mathrm{~nm})$ LED lights were applied as light stimuli for LamPP. Red (600 nm) LED light was used as a background light while LamPP-worms were fed all-trans retinal. Avoidance response probabilities were calculated by the ratio or the number of responded worms to five worms examined.

Generation of chimeric mutants of opsins. Chimeric mutants of MosOpn3 and LamPP were generated by replacing cytoplasmic regions of hamster $\$ 2$-adrenergic receptor (B2AR) and human $\alpha 1$-adrenergic receptor ( $\alpha 1 \mathrm{AR})$ by combining DNA fragments of each region with PCR. The cytoplasmic regions were determined according to previous reports $^{5,50}$ and applied to MosOpn3 and LamPP based on the alignment including bovine rhodopsin.

Bioluminescent reporter assays for $\mathrm{Ca}^{2+}$ and cAMP. The intracellular cAMP and $\mathrm{Ca}^{2+}$ levels in opsin-expressing HEK293S cells were measured using the GloSensor cAMP assay and the aequorin assay, respectively, as described previously ${ }^{22,26,67,68}$. The pGloSensor-22F cAMP plasmid (Promega) was used for the GloSensor cAMP assay. The 
wild type aequorin obtained by introducing two reverse mutations into the plasmid [pcDNA3.1+/mit-2mutAEQ] (Addgene \#45539) ${ }^{69}$ was used for the aequorin assay. A broad band green LED light and narrow band violet (410 $\mathrm{nm})$ and blue (430 nm) LED lights were applied for $5 \mathrm{sec}$ in the GloSensor cAMP assay and for $1 \mathrm{sec}$ in the aequorin assay as light stimuli.

Acknowledgements. We thank Robert J. Lucas (The University of Manchester) for providing the promoter of unc-17. This work was supported in part by grants-in-aid for Scientific Research from the Japanese Ministry of Education, Science, Sports and Culture 18H02482, 20K21433, $21 \mathrm{H} 00435$ (to M.K.), 15H05777, 20K21434 (to A.T.); Japan Science and Technology Agency (JST) Precursory Research for Embryonic Science and Technology (PRESTO) Grant JPMJPR13A2 (to M.K.) and JST Core Research for Evolutional Science and Technology (CREST) Grant JPMJCR1753 (to A.T.).

Author Contributions. M.K. and A.T. conceived and designed the study. M.K., B.S., T.N., L.S., S.W., S.K., and E.K.-N. carried out the experiments and analysed the data. M.K. and A.T. wrote and edited the paper. 


\section{References}

1. Bockaert J, Pin JP. Molecular tinkering of G protein-coupled receptors: an evolutionary success. EMBO J 18, 1723-1729 (1999).

2. Pierce KL, Premont RT, Lefkowitz RJ. Seven-transmembrane receptors. Nat Rev Mol Cell Biol 3, 639-650 (2002).

3. Lagerstrom MC, Schioth HB. Structural diversity of G protein-coupled receptors and significance for drug discovery. Nat Rev Drug Discov 7, 339-357 (2008).

4. Koyanagi M, Terakita A. Diversity of animal opsin-based pigments and their optogenetic potential. Biochim Biophys Acta 1837, 710-716 (2014).

5. Airan RD, Thompson KR, Fenno LE, Bernstein H, Deisseroth K. Temporally precise in vivo control of intracellular signalling. Nature 458, 1025-1029 (2009).

6. Cao P, et al. Light-sensitive coupling of rhodopsin and melanopsin to G(i/o) and G(q) signal transduction in Caenorhabditis elegans. FASEB J 26, 480-491 (2012).

7. Karunarathne WK, Giri L, Kalyanaraman V, Gautam N. Optically triggering spatiotemporally confined GPCR activity in a cell and programming neurite initiation and extension. Proc Natl Acad Sci U S A 110, E1565-1574 (2013).

8. Koyanagi M, Saito T, Wada S, Nagata T, Kawano-Yamashita E, Terakita A. Optogenetic Potentials of Diverse Animal Opsins: Parapinopsin, Peropsin, LWS Bistable Opsin. Adv Exp Med Biol 1293, 141-151 (2021). 
9. Tsukamoto H, Terakita A. Diversity and functional properties of bistable pigments.

Photochem Photobiol Sci 9, 1435-1443 (2010).

10. Terakita A, Tsukamoto H, Koyanagi M, Sugahara M, Yamashita T, Shichida Y.

Expression and comparative characterization of Gq-coupled invertebrate visual

pigments and melanopsin. J Neurochem 105, 883-890 (2008).

11. Nagata T, et al. Depth perception from image defocus in a jumping spider. Science

335, 469-471 (2012).

12. Nagata T, et al. The counterion-retinylidene Schiff base interaction of an

invertebrate rhodopsin rearranges upon light activation. Commun Biol 2, 180

(2019).

13. Saito T, Koyanagi M, Sugihara T, Nagata T, Arikawa K, Terakita A. Spectral

tuning mediated by helix III in butterfly long wavelength-sensitive visual opsins

revealed by heterologous action spectroscopy. Zoological Lett 5, 35 (2019).

14. Ye H, Daoud-El Baba M, Peng RW, Fussenegger M. A synthetic optogenetic

transcription device enhances blood-glucose homeostasis in mice. Science 332,

1565-1568 (2011).

15. Tsunematsu T, Tanaka KF, Yamanaka A, Koizumi A. Ectopic expression of melanopsin in orexin/hypocretin neurons enables control of wakefulness of mice 
in vivo by blue light. Neurosci Res 75, 23-28 (2013).

16. van Wyk M, Pielecka-Fortuna J, Lowel S, Kleinlogel S. Restoring the ON Switch in Blind Retinas: Opto-mGluR6, a Next-Generation, Cell-Tailored Optogenetic Tool. PLoS Biol 13, e1002143 (2015).

17. Koyanagi M, et al. Bistable UV pigment in the lamprey pineal. Proc Natl Acad Sci U S A 101, 6687-6691 (2004).

18. Tsukamoto H, Terakita A, Shichida Y. A rhodopsin exhibiting binding ability to agonist all-trans-retinal. Proc Natl Acad Sci U S A 102, 6303-6308 (2005).

19. Koyanagi M, Kubokawa K, Tsukamoto H, Shichida Y, Terakita A.

Cephalochordate melanopsin: evolutionary linkage between invertebrate visual cells and vertebrate photosensitive retinal ganglion cells. Curr Biol 15, 1065-1069 (2005).

20. Koyanagi M, Takano K, Tsukamoto H, Ohtsu K, Tokunaga F, Terakita A. Jellyfish vision starts with cAMP signaling mediated by opsin-G(s) cascade. Proc Natl Acad Sci U S A 105, 15576-15580 (2008).

21. Nagata T, Koyanagi M, Tsukamoto H, Terakita A. Identification and characterization of a protostome homologue of peropsin from a jumping spider. $J$ Comp Physiol A Neuroethol Sens Neural Behav Physiol 196, 51-59 (2010). 
22. Koyanagi M, Takada E, Nagata T, Tsukamoto H, Terakita A. Homologs of vertebrate Opn3 potentially serve as a light sensor in nonphotoreceptive tissue.

Proc Natl Acad Sci U S A 110, 4998-5003 (2013).

23. Koyanagi M, et al. Diversification of non-visual photopigment parapinopsin in spectral sensitivity for diverse pineal functions. BMC Biol 13, (2015).

24. He XJ, Banghart MR. It's lights out for presynaptic terminals. Neuron 109, 1755-1757 (2021).

25. Blackshaw S, Snyder SH. Encephalopsin: a novel mammalian extraretinal opsin discretely localized in the brain. J Neurosci 19, 3681-3690 (1999).

26. Sugihara T, Nagata T, Mason B, Koyanagi M, Terakita A. Absorption Characteristics of Vertebrate Non-Visual Opsin, Opn3. PLoS ONE 11, e0161215 (2016).

27. Terakita A, Nagata T. Functional properties of opsins and their contribution to light-sensing physiology. Zoolog Sci 31, 653-659 (2014).

28. Mahn M, et al. Efficient optogenetic silencing of neurotransmitter release with a mosquito rhodopsin. Neuron 109, 1621-1635 e1628 (2021).

29. Blackshaw S, Snyder SH. Parapinopsin, a novel catfish opsin localized to the parapineal organ, defines a new gene family. J Neurosci 17, 8083-8092 (1997). 
30. Wada S, Kawano-Yamashita E, Koyanagi M, Terakita A. Expression of

UV-sensitive parapinopsin in the iguana parietal eyes and its implication in

UV-sensitivity in vertebrate pineal-related organs. PLoS ONE 7, e39003 (2012).

31. Koyanagi M, Kawano-Yamashita E, Wada S, Terakita A. Vertebrate Bistable

Pigment Parapinopsin: Implications for Emergence of Visual Signaling and

Neofunctionalization of Non-visual Pigment. Front Ecol Evol 5, 23 (2017).

32. Terakita A, Koyanagi M, Tsukamoto H, Yamashita T, Miyata T, Shichida Y.

Counterion displacement in the molecular evolution of the rhodopsin family. Nat

Struct Mol Biol 11, 284-289 (2004).

33. Kawano-Yamashita E, Koyanagi M, Wada S, Tsukamoto H, Nagata T, Terakita A.

Activation of Transducin by Bistable Pigment Parapinopsin in the Pineal Organ of

Lower Vertebrates. PLoS ONE 10, (2015).

34. Wada S, Kawano-Yamashita E, Sugihara T, Tamotsu S, Koyanagi M, Terakita A.

Insights into the evolutionary origin of the pineal color discrimination mechanism

from the river lamprey. BMC Biol 19, 188 (2021).

35. Wada S, et al. Color opponency with a single kind of bistable opsin in the zebrafish pineal organ. Proc Natl Acad Sci U S A 115, 11310-11315 (2018).

36. Eickelbeck D, et al. Lamprey Parapinopsin ("UVLamP"): a Bistable UV-Sensitive 
Optogenetic Switch for Ultrafast Control of GPCR Pathways. Chembiochem 21, 612-617 (2020).

37. Rodgers J, et al. Using a bistable animal opsin for switchable and scalable optogenetic inhibition of neurons. EMBO Rep 22, e51866 (2021).

38. Copits BA, et al. A photoswitchable GPCR-based opsin for presynaptic inhibition. Neuron 109, 1791-1809 e1711 (2021).

39. Nagel G, Brauner M, Liewald JF, Adeishvili N, Bamberg E, Gottschalk A. Light activation of channelrhodopsin-2 in excitable cells of Caenorhabditis elegans triggers rapid behavioral responses. Curr Biol 15, 2279-2284 (2005).

40. Roayaie K, Crump JG, Sagasti A, Bargmann CI. The G alpha protein ODR-3 mediates olfactory and nociceptive function and controls cilium morphogenesis in C. elegans olfactory neurons. Neuron 20, 55-67 (1998).

41. Hilliard MA, Bergamasco C, Arbucci S, Plasterk RH, Bazzicalupo P. Worms taste bitter: ASH neurons, QUI-1, GPA-3 and ODR-3 mediate quinine avoidance in Caenorhabditis elegans. EMBO J 23, 1101-1111 (2004).

42. Hori S, Oda S, Suehiro Y, Iino Y, Mitani S. OFF-responses of interneurons optimize avoidance behaviors depending on stimulus strength via electrical synapses. PLoS Genet 14, e1007477 (2018). 
43. Edwards SL, et al. A novel molecular solution for ultraviolet light detection in Caenorhabditis elegans. PLoS Biol 6, e198 (2008).

44. Gong J, et al. The C. elegans Taste Receptor Homolog LITE-1 Is a Photoreceptor. Cell 167, 1252-1263 e1210 (2016).

45. Terakita A, Yamashita T, Nimbari N, Kojima D, Shichida Y. Functional interaction between bovine rhodopsin and G protein transducin. J Biol Chem 277, 40-46 (2002).

46. Liu J, Conklin BR, Blin N, Yun J, Wess J. Identification of a receptor/G-protein contact site critical for signaling specificity and G-protein activation. Proc Natl Acad Sci U S A 92, 11642-11646 (1995).

47. Wess J. G-protein-coupled receptors: molecular mechanisms involved in receptor activation and selectivity of G-protein recognition. FASEB J 11, 346-354 (1997).

48. Eason MG, Liggett SB. Chimeric mutagenesis of putative G-protein coupling domains of the alpha2A-adrenergic receptor. Localization of two redundant and fully competent gi coupling domains. J Biol Chem 271, 12826-12832 (1996).

49. Yamashita T, Terakita A, Shichida Y. Distinct roles of the second and third cytoplasmic loops of bovine rhodopsin in G protein activation. J Biol Chem $\mathbf{2 7 5}$, 34272-34279 (2000). 
50. Kim JM, Hwa J, Garriga P, Reeves PJ, RajBhandary UL, Khorana HG.

Light-driven activation of beta 2 -adrenergic receptor signaling by a chimeric rhodopsin containing the beta 2-adrenergic receptor cytoplasmic loops.

Biochemistry 44, 2284-2292 (2005).

51. Palczewski K. G protein-coupled receptor rhodopsin. Annu Rev Biochem $\mathbf{7 5}$, $743-767$ (2006).

52. Sunahara RK, Dessauer CW, Gilman AG. Complexity and diversity of mammalian adenylyl cyclases. Annu Rev Pharmacol Toxicol 36, 461-480 (1996).

53. Willoughby D, Cooper DM. Organization and $\mathrm{Ca} 2+$ regulation of adenylyl cyclases in cAMP microdomains. Physiol Rev 87, 965-1010 (2007).

54. Sadana R, Dessauer CW. Physiological roles for G protein-regulated adenylyl cyclase isoforms: insights from knockout and overexpression studies.

Neurosignals 17, 5-22 (2009).

55. Alfonso A, Grundahl K, Duerr JS, Han HP, Rand JB. The Caenorhabditis elegans unc-17 gene: a putative vesicular acetylcholine transporter. Science 261, 617-619 (1993).

56. Johnson JR, et al. Ethanol Stimulates Locomotion via a Galphas-Signaling Pathway in IL2 Neurons in Caenorhabditis elegans. Genetics 207, 1023-1039 
(2017).

57. Boyden ES. A history of optogenetics: the development of tools for controlling brain circuits with light. F1000 Biol Rep 3, 11 (2011).

58. Deisseroth K. Optogenetics. Nat Methods 8, 26-29 (2011).

59. Hegemann P, Nagel G. From channelrhodopsins to optogenetics. EMBO Mol Med 5, 173-176 (2013).

60. Deisseroth K, Hegemann P. The form and function of channelrhodopsin. Science 357, eaan5544 (2017).

61. Husson SJ, Liewald JF, Schultheis C, Stirman JN, Lu H, Gottschalk A. Microbial light-activatable proton pumps as neuronal inhibitors to functionally dissect neuronal networks in C. elegans. PLoS ONE 7, e40937 (2012).

62. Armbruster BN, Li X, Pausch MH, Herlitze S, Roth BL. Evolving the lock to fit the key to create a family of G protein-coupled receptors potently activated by an inert ligand. Proc Natl Acad Sci U S A 104, 5163-5168 (2007).

63. Conklin BR, et al. Engineering GPCR signaling pathways with RASSLs. Nat Methods 5, 673-678 (2008).

64. Urban DJ, Roth BL. DREADDs (designer receptors exclusively activated by designer drugs): chemogenetic tools with therapeutic utility. Annu Rev Pharmacol 
Toxicol 55, 399-417 (2015).

65. Macosko EZ, et al. A hub-and-spoke circuit drives pheromone attraction and social behaviour in C. elegans. Nature 458, 1171-1175 (2009).

66. Brenner S. The genetics of Caenorhabditis elegans. Genetics 77, 71-94 (1974).

67. Bailes HJ, Lucas RJ. Human melanopsin forms a pigment maximally sensitive to blue light (lambdamax approximately $479 \mathrm{~nm}$ ) supporting activation of G(q/11) and G(i/o) signalling cascades. Proc Biol Sci 280, 20122987 (2013).

68. Nagata T, Koyanagi M, Lucas R, Terakita A. An all-trans-retinal-binding opsin peropsin as a potential dark-active and light-inactivated $\mathrm{G}$ protein-coupled receptor. Sci Rep 8, 3535 (2018).

69. de la Fuente S, Fonteriz RI, de la Cruz PJ, Montero M, Alvarez J. Mitochondrial free $[\mathrm{Ca}(2+)]$ dynamics measured with a novel low- $\mathrm{Ca}(2+)$ affinity aequorin probe. Biochem J 445, 371-376 (2012). 


\section{Figure legends}

Fig. 1. Examination of the functionality of MosOpn3 in ASH neurons of C. elegans.

a The construction for introducing MosOpn3 with EGFP under the sra-6 promoter. A selection marker mCherry was also introduced under the promotor of myo-2. b GPCR signaling in ASH neurons. Activation of ODR-3, a Gi-like protein eventually causes avoidance behavior of $C$. elegans. c Experimental setup. Worms were continuously monitored with infrared light. Light stimuli were supplied using a dichroic mirror and the intensities were regulated with ND filters.

Fig. 2. Functionality of MosOpn3 in ASH neurons of C. elegans.

a Snapshot images at $0.7,1.6,2.5,3.4,4.3$ and $5.2 \mathrm{~s}$ after recording start, showing light-induced avoidance responses of MosOpn3-expressing Tg worms in the presence of 11-cis (upper) and all-trans (lower) retinal. The horizontal bar and mCherry signals emitted by the light illumination indicate the timing of white light stimulus. Dotted lines indicated the position of the frontal tip of worms when they stopped moving forward. b, $\mathbf{c}$ White light intensity-response probability relationships for MosOpn3-worms (magenta squares and curve) and BovRh-worms (green squares and curve) in the minimum necessary amount 11-cis retinal $(\mathbf{b}, \mathrm{n}=6)$ and all-trans retinal $(\mathbf{c}, \mathrm{n}=3)$. Both Tg worms 
exhibited a similar light sensitivity in the case of 11-cis retinal whereas only

MosOpn3-worms responded to light under the presence of all-trans retinal. The light

intensity-response probability relationship for MosOpn3/11-worms is also indicated in (c)

for comparison (black open squares and dashed curve), showing a similar performance of

MosOpn3/11- and MosOpn3/AT-worms in the light-induced avoidance behavior. d, e

White light intensity-response probability relationships for MosOpn3/11-worms (black

triangles) and MosOpn3/AT-worms (blue triangles) under the presence of 1/1000 (d, n=3)

and 1/10000 (e, n=3) amount of the standard amount of retinal. The data for

MosOpn3/AT-worms under the minimum necessary amount retinal were also indicated

for comparison (magenta filled squares and dashed curve). $\mathrm{I}_{0}=\sim 0.8 \mathrm{~mW} / \mathrm{mm}^{2}$ (b-e).

\section{Fig. 3. Engineering MosOpn3-based tools to activate Gs or Gq-type G protein.}

a GloSensor cAMP assay with HEK293 cells expressing various MosOpn3-ß2AR

chimeras, showing light-induced increases of intracellular cAMP levels. MosOpn3

chimera containing the third cytoplasmic loop of jellyfish Gs-coupled opsin

(MosOpn3-JelOpiL3) was also shown for a comparison. The light-induced cAMP decease

in HEK293 cells expressing MosOpn3 WT was shown in inset. Vertical lines indicate

light illumination for $5 \mathrm{sec}$. b Aequorin $\mathrm{Ca}^{2+}$ assay with HEK293 cells expressing 
MosOpn3- $\alpha 1$ ARiL3 chimeras having varied length of third cytoplasmic loop region of

$\alpha 1 \mathrm{AR}$, showing light-induced increases of intracellular $\mathrm{Ca}^{2+}$ levels (left). Light-induced

changes of intracellular cAMP levels of HEK293 cells expressing the MosOpn3- $\alpha 1$ AR

chimeras were also shown (right). Note that other MosOpn3- $\alpha 1$ AR chimeras did not show

significant improvement of light-induced $\mathrm{Ca}^{2+}$ elevation. Vertical lines indicate light

illumination for $1 \mathrm{sec}$ (left), and $5 \mathrm{sec}$ (right).

Fig. 4. Color-dependent manipulation of Tg C. elegans expressing LamPP in

\section{cholinergic motor neurons.}

a The construction for introducing LamPP with EGFP under the unc-17 promoter. b

Schematic drawing of the relationship between status of LamPP and C. elegans behavior

caused by violet and green light illumination. LamPP* indicates the active state of LamPP.

c Snapshot images of the behavior of $C$. elegans expressing LamPP in cholinergic neurons

in the dark, after violet illumination and subsequent green light illumination, showing

moving forward, coiling and restart moving, respectively. See also Supplementary Movie

2. d Quantitative evaluation of violet light-induced coiling and subsequent green

light-induced recovering of LamPP-worms (n=5). LamPP-worms fed E. coli without

retinal (grey circles), with 11-cis retinal (magenta circles) in the dark and with all-trans 
retinal under the red light (blue circles) were illuminated with violet light for $5 \mathrm{sec}$ (violet

line), kept in the dark for $1 \mathrm{~min}$ and illuminated with green light for $30 \mathrm{sec}$ (green line). $\mathbf{e}$

Violet light-induced coiling of LamPP-worms fed 11-cis retinal sustained for 30 min after

turn-off of the violet light. All worms exhibited immobility and/or coiling for $30 \mathrm{~min}$.

Upon green illumination, all worms restarted moving.

Fig. 5. Color dependent manipulation of cAMP level by LamPP-B2ARiL3 through

the activation of Gs-type G protein measured by GloSensor cAMP assay.

a Violet light illumination increased intracellular cAMP levels in HEK293 cells

expressing LamPP-ß2ARiL3 and subsequent green light illumination decreased the cAMP

level to the base level, which occurred repeatedly. b Blue light illumination generated a

particular cAMP level. The cAMP levels generated by (i) 1st illumination, (ii) the

subsequent illumination, (iii) the illumination after violet light illumination, (iv) the

subsequent illumination, (v) the illumination after green light illumination and (vi) the

subsequent illumination of blue light were almost constant, showing color-dependent

cAMP clamping. Vertical lines indicate light illumination for $5 \mathrm{sec}$. 
a



Avoidance behavior

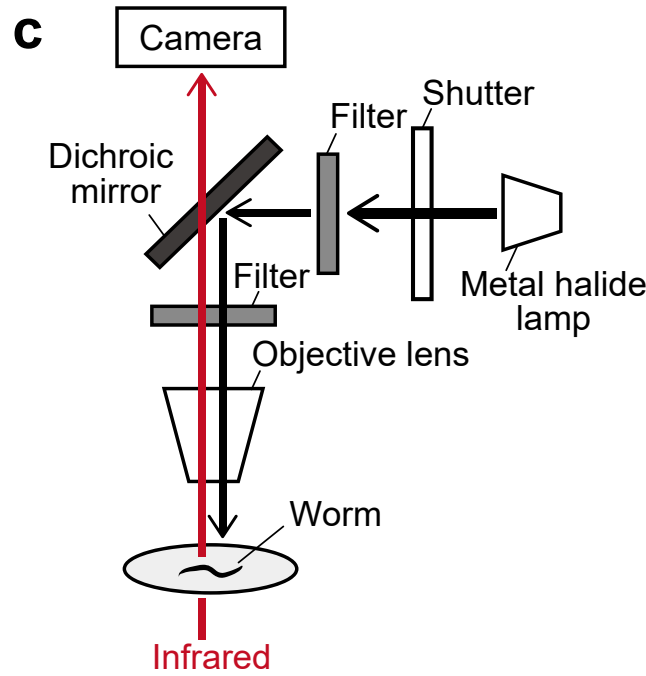

b

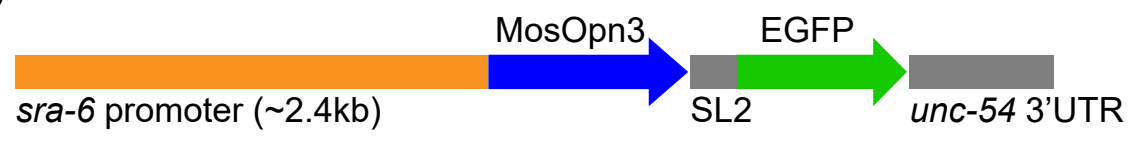

mCherry

myo-2 promoter ( 1.3kb) unc-86 3'UTR

Fig. 1 Koyanagi et al. 
bioRxiv preprint doi: https://doi.org/10.1101/2022.02.07.479375; this version posted February 8, 2022. The copyright holder for this preprint (which was not certified by peer review) is the author/funder. All rights reserved. No reuse allowed without permission.

\section{a}

11-cis

retinal

all-trans retinal

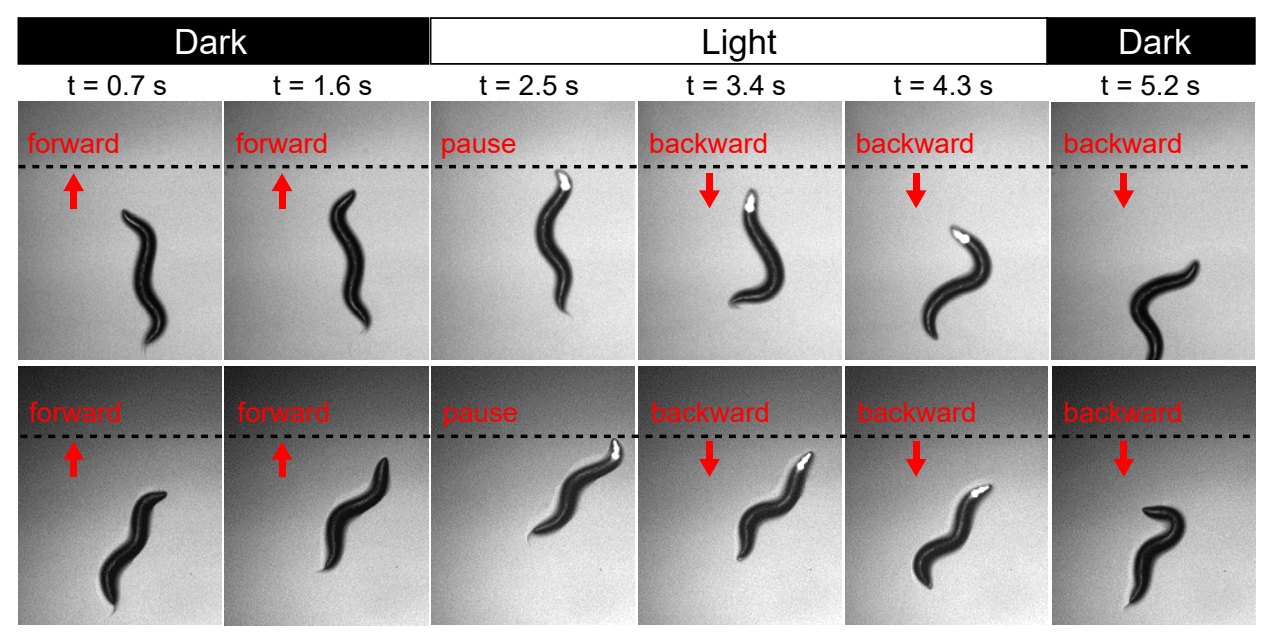

b

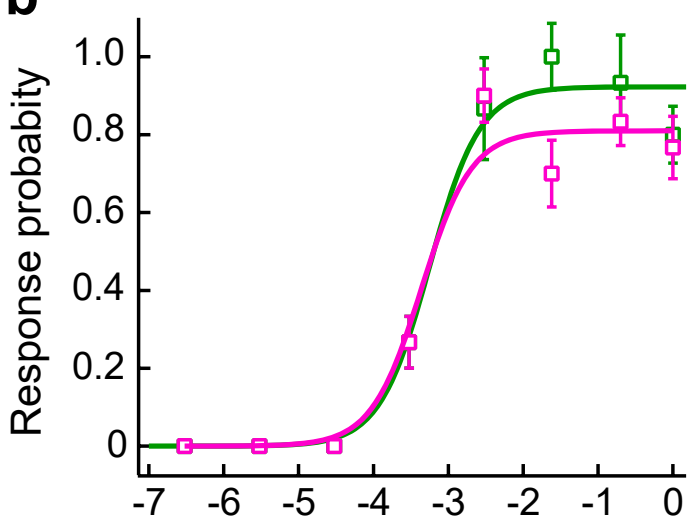

Light intensity $\left(\log 1 / I_{0}\right)$

d

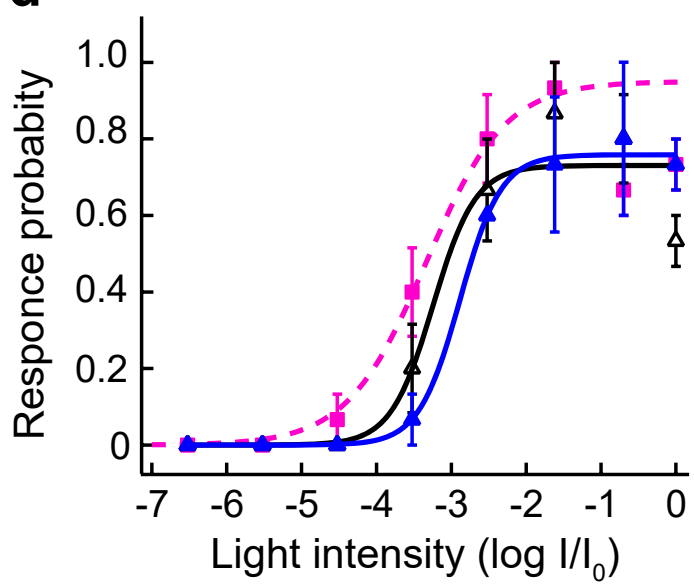

C

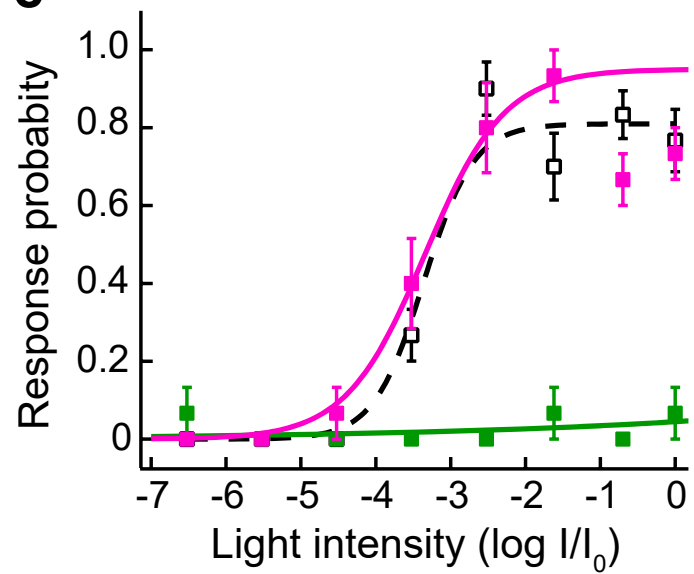

e

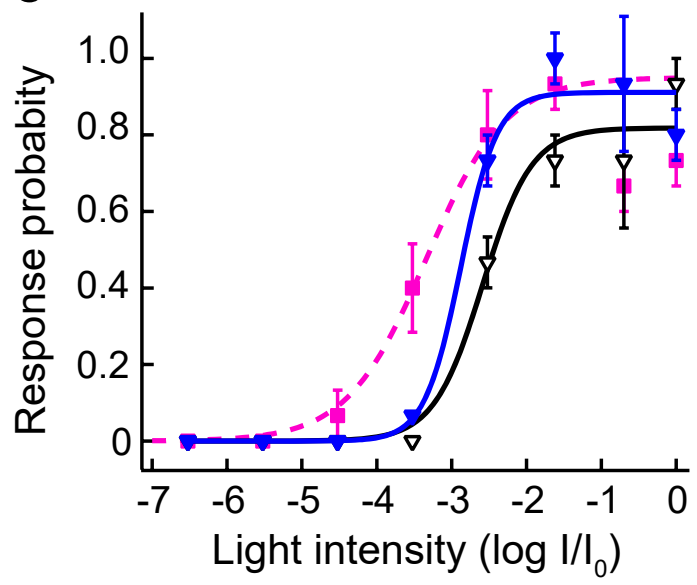

Fig. 2 Koyanagi et al. 
bioRxiv preprint doi: https://doi.org/10.1101/2022.02.07.479375; this version posted February 8, 2022. The copyright holder for this preprint (which was not certified by peer review) is the author/funder. All rights reserved. No reuse allowed without permission.

a

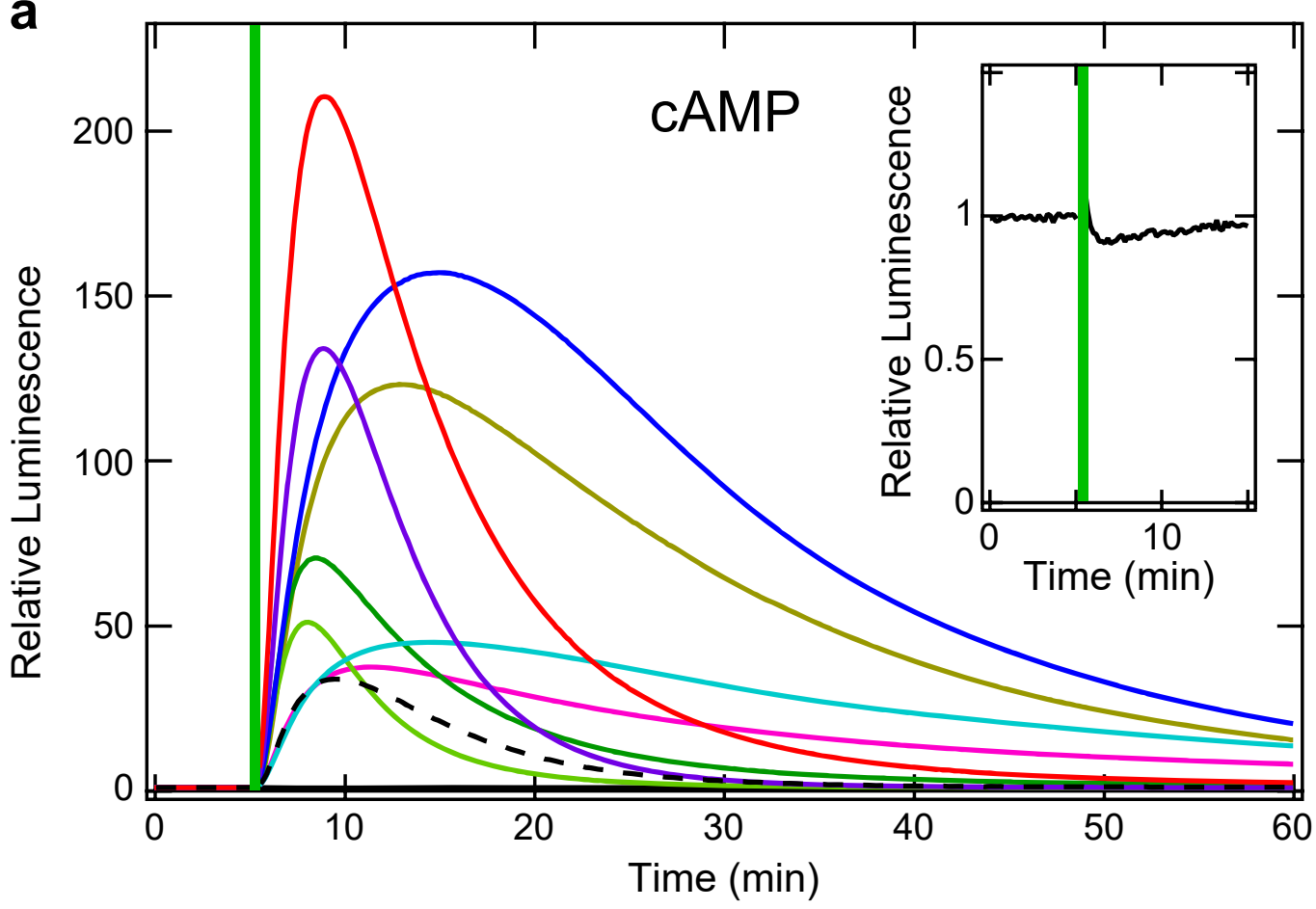

- MosOpn3-WT

- MosOpn3-ß2ARiL3

- MosOpn3-32ARiL3C

MosOpn3-32ARiL13

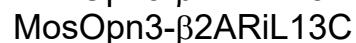

MosOpn3-ß2ARiL23

MosOpn3-32ARiL23C

MosOpn3-B2ARiL123

MosOpn3-ß2ARiL123C

- - MosOpn3-JelOpiL3
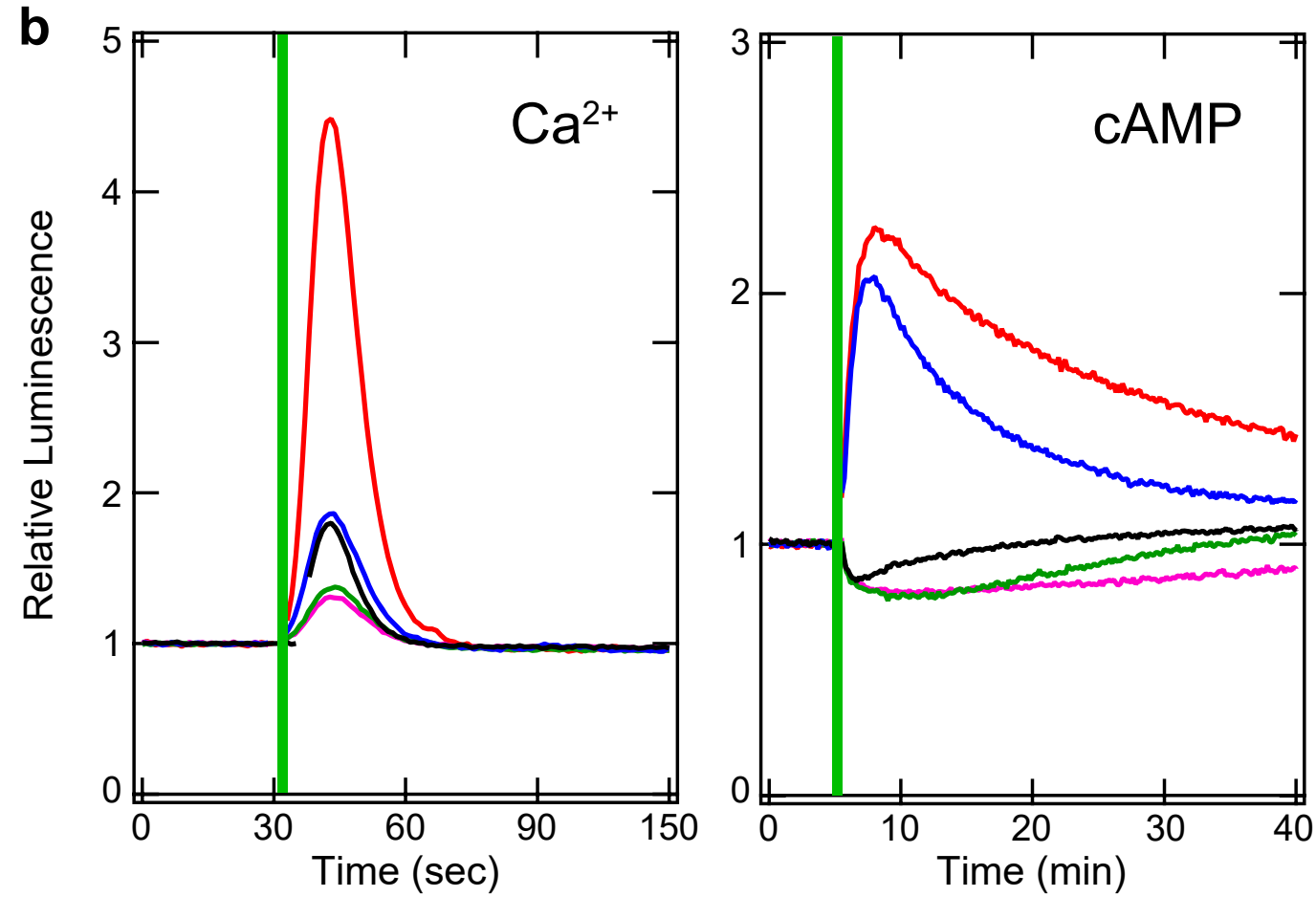

MosOpn3

MosOpn3- $\alpha 1$ ARiL3

MosOpn3- $\alpha 1$ ARiL3(-2/0)

MosOpn3- $\alpha 1$ ARiL3(-2/+2)

MosOpn3- $\alpha 1$ ARiL3(-2/+4)

Fig. 3 Koyanagi et al. 
bioRxiv preprint doi: https://doi.org/10.1101/2022.02.07.479375; this version posted February 8, 2022. The copyright holder for this preprint (which was not certified by peer review) is the author/funder. All rights reserved. No reuse allowed without permission.

a

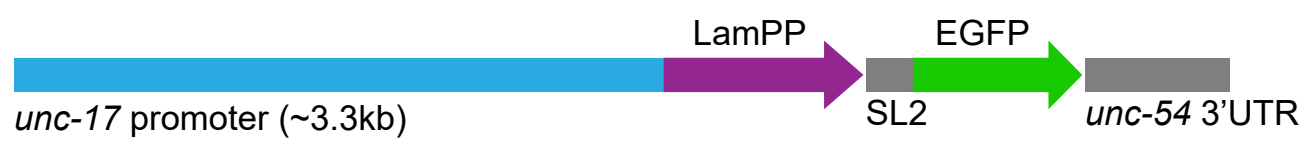

b

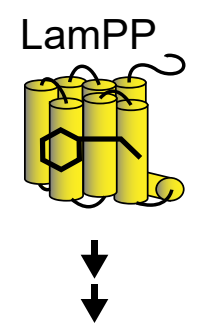

Normal

Ach release

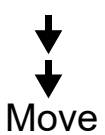

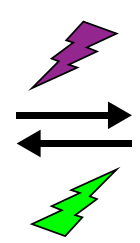



Abnormal

Ach release

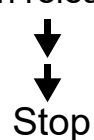

C
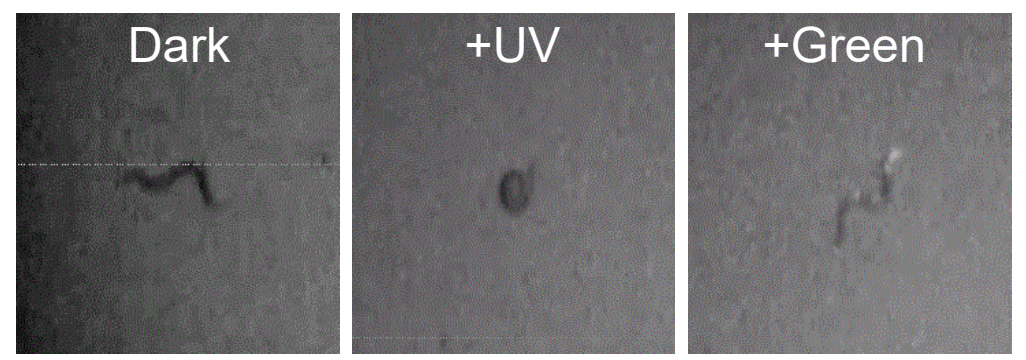

d

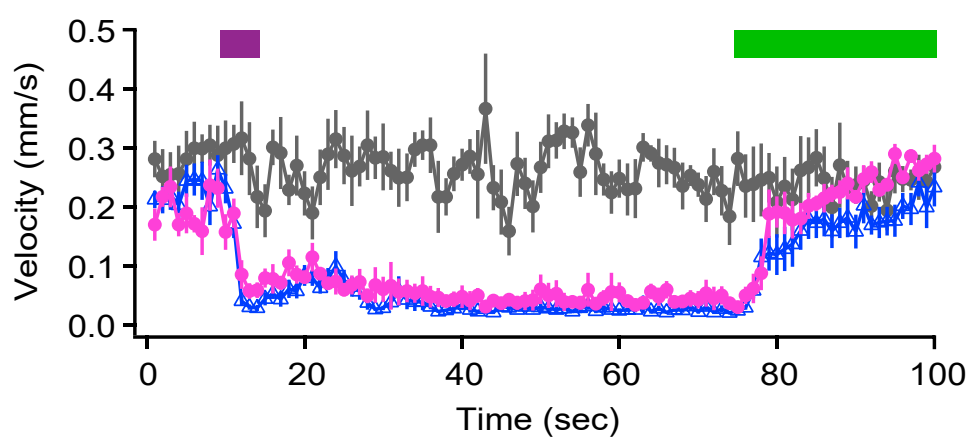

e

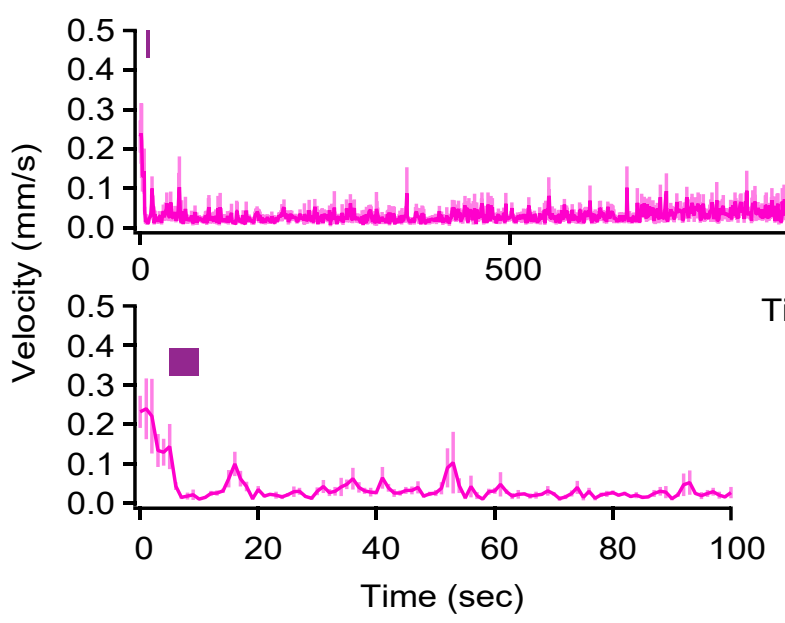

1000

Time $(\mathrm{sec})$
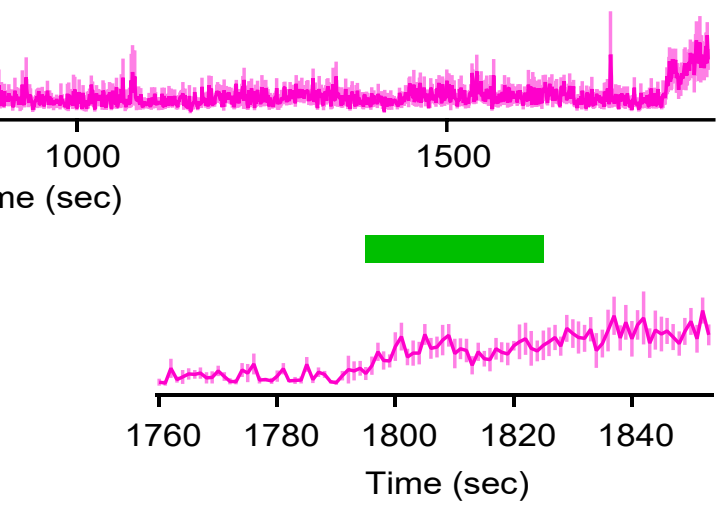

Fig. 4 Koyanagi et al. 

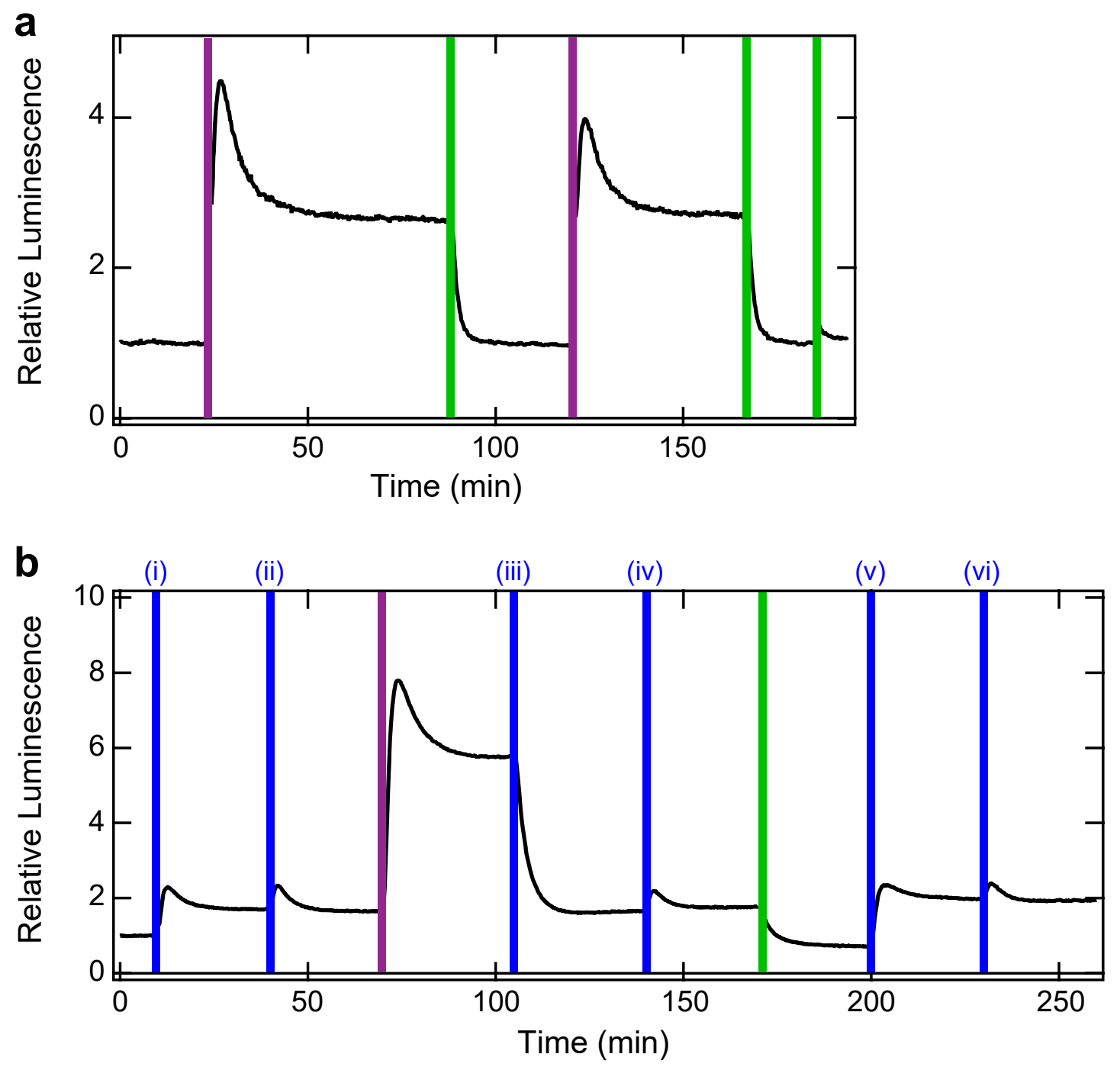

Fig. 5 Koyanagi et al. 